TRANSACTIONS OF THE

AMERICAN MATHEMATICAL SOCIETY

Volume 353, Number 12, Pages 5165-5184

S 0002-9947(01)02868-9

Article electronically published on June 14, 2001

\title{
MAXIMAL SEMIGROUPS IN SEMI-SIMPLE LIE GROUPS
}

\author{
LUIZ A. B. SAN MARTIN
}

\begin{abstract}
The maximal semigroups with nonempty interior in a semi-simple Lie group with finite center are characterized as compression semigroups of subsets in the flag manifolds of the group. For this purpose a convexity theory, called here $\mathcal{B}$-convexity, based on the open Bruhat cells is developed. It turns out that a semigroup with nonempty interior is maximal if and only if it is the compression semigroup of the interior of a $\mathcal{B}$-convex set.
\end{abstract}

\section{INTRODUCTION}

The purpose of this paper is to characterize the maximal semigroups with nonempty interior in semi-simple Lie groups with finite center. The main result is Theorem 5.4 which gives a precise description of the maximal semigroups through their actions on the flag manifolds of the group.

When studying semigroups embedded into groups many different questions have a natural formulation and solution by means of the knowledge of the maximal semigroups on a specific group. This makes the problem of determining the maximal semigroups one of the major ones in the theory of semigroups. For semigroups in Lie groups J. Lawson [8], appealing to the Levi decomposition of a Lie algebra, divided the task of classifying - or at least understanding - the maximal semigroups, by considering two main classes namely the semigroups of solvable type and those of semi-simple type, according to the kind of Lie group containing them. In order to understand the maximal semigroups in a general Lie group $G$ it is required to have a classification of these two types, and then mix them up in $G$. In [8] Lawson himself provided a classification of the maximal semigroups with nonempty interior in solvable groups: There is a one-to-one correspondence between the maximal subsemigroups and the half-spaces in the Lie algebra bounded by a hyperplane subalgebra. Thus for solvable groups the maximal semigroups have an algebraic nature. This classification is extended to compact extensions of solvable groups in [8] (see also Hilgert and Neeb [6]), and to semigroups in lattices of solvable groups (see do Rocio and San Martin [11]).

In a semi-simple Lie group $G$ with finite center it was proved in San Martin and Tonelli [14] that any maximal semigroup $S \subset G$ with nonempty interior is a compression semigroup of a subset $C$ of one of the minimal flag manifolds of $G$ :

$$
S=S_{C}=\{g \in G: g C \subset C\} .
$$

Received by the editors March 18, 1999 and, in revised form, March 29, 2001.

2000 Mathematics Subject Classification. Primary 20M20, 22E20, 22F30.

Key words and phrases. Semigroups, semi-simple Lie groups, flag manifolds, convexity. Research partially supported by CNPq grant $n^{\circ} 301060 / 94-0$. 
However, in order to have a complete picture of the maximal semigroups in $G$, it is required to find the appropriate family of sets $C$ such that $S_{C}$ is indeed maximal. In 14 this was done only for the real rank one simple Lie groups. Here we provide the appropriate sets for general semi-simple groups, generalizing the rank one case. The approach is through a convexity theory for subsets of the flag manifolds. Precisely, we say that a subset of a flag manifold is $\mathcal{B}$-convex if it is the intersection of all open Bruhat cells containing it. This notion of convexity is formally defined by a convex hull operator on subsets. This operator, in turn, comes from a duality operator mapping subsets of a flag manifold into subsets of the dual flag manifold. Having settled this convexity theory we prove that a semigroup with nonvoid interior in $G$ is maximal if and only if it is the compression semigroup of the interior of a $\mathcal{B}$ convex set in a minimal flag manifold. We point out that the same characterization also holds for partial maximal semigroups in the following sense: In [14 it was established that there are different classes of semigroups with nonempty interior in a semi-simple Lie group, namely, one class for each flag manifold of the group (see Section 4 below). A partial maximal semigroup (called $\Theta$-maximal) is a semigroup which is maximal within the class given by a flag manifold. These partial maximal semigroups are also described by compression of $\mathcal{B}$-convex sets, but in this case on flag manifolds different from the minimal ones.

In some simple examples, the $\mathcal{B}$-convex sets are rather arbitrary subsets. For instance in a real rank one group any nonempty subset of the flag manifold is $\mathcal{B}$-convex. Although in general the $\mathcal{B}$-convex sets are not as trivial as that, their defining conditions are quite faint, reflecting in the existence of a great profusion of nonconjugate maximal semigroups. This richness is in the realm of the structure of semi-simple Lie groups. It can be revealed with a further understanding of the $\mathcal{B}$-convex sets.

\section{Preliminaries}

In this section we give the notations and basic facts about semi-simple Lie algebras and the associated flag manifolds which are used throughout the paper.

Let $\mathfrak{g}$ be a noncompact semi-simple Lie algebra. We make the following standard choices in $\mathfrak{g}$. Let $\theta$ be a Cartan involution of $\mathfrak{g}$ and $\mathfrak{g}=\mathfrak{k} \oplus \mathfrak{s}$ the associated Cartan decomposition with $\mathfrak{k}$ standing for the subalgebra of $\theta$-fixed points. Select a maximal abelian subalgebra $\mathfrak{a} \subset \mathfrak{s}$ and let $\Pi$ be the set of restricted roots of the pair $(\mathfrak{g}, \mathfrak{a})$. For a root $\alpha \in \Pi$, its root space is denoted by $\mathfrak{g}_{\alpha}$. Choose a simple system of roots $\Sigma \subset \Pi$ and denote by $\Pi^{+}$the set of positive roots spanned by $\Sigma$. We let $\mathfrak{a}^{+}$stand for the Weyl chamber associated to $\Pi^{+}$and

$$
\mathfrak{n}^{ \pm}=\sum_{\alpha \in \Pi^{ \pm}} \mathfrak{g}_{\alpha}
$$

for the nilpotent subalgebras associated with $\Pi^{+}$and $\Pi^{-}=-\Pi^{+}$, respectively. Denote by $\mathfrak{m}$ the centralizer of $\mathfrak{a}$ in $\mathfrak{k}$.

The subalgebra $\mathfrak{p}=\mathfrak{m} \oplus \mathfrak{a} \oplus \mathfrak{n}^{+}$is the standard minimal parabolic subalgebra of $\mathfrak{g}$. More generally, if $\Theta \neq \Sigma$ is a subset of $\Sigma$ we denote by $\mathfrak{p}_{\Theta}$ the parabolic subalgebra

$$
\mathfrak{p}_{\Theta}=\mathfrak{n}^{-}(\Theta) \oplus \mathfrak{p} .
$$

Here $\mathfrak{n}^{-}(\Theta)$ is the subalgebra spanned by the root spaces $\mathfrak{g}_{-\alpha}, \alpha \in\langle\Theta\rangle$, where $\langle\Theta\rangle$ is the set of positive roots generated by $\Theta$. Of course, $\mathfrak{p}=\mathfrak{p}_{\emptyset}$. 
Let $G$ be a Lie group with Lie algebra $\mathfrak{g}$. We assume always that $G$ has finite center. In this case the subgroup $K=\exp \mathfrak{k}$ is compact. For $g \in G$ and $X \in \mathfrak{g}$ we put $g \cdot X$ for the adjoint action of $g$ in $X$. The parabolic subgroup $P_{\Theta}$ is the normalizer of $\mathfrak{p}_{\Theta}$ in $G$ :

$$
P_{\Theta}=\left\{g \in G: g \cdot \mathfrak{p}_{\Theta}=\mathfrak{p}_{\Theta}\right\} .
$$

Its Lie algebra is $\mathfrak{p}_{\Theta}$. The flag manifold $\mathbb{B}_{\Theta}=G / P_{\Theta}$ is realized as the set $\left\{g \cdot \mathfrak{p}_{\Theta}\right.$ : $g \in G\}$ of parabolic subalgebras conjugate to $\mathfrak{p}_{\Theta}$. Alternatively, let $\mathfrak{n}_{\Theta}^{+}$stand for the nilpotent radical (nilradical) of $\mathfrak{p}_{\Theta}$. Explicitly, $\mathfrak{n}_{\Theta}^{+}=\sum_{\alpha} \mathfrak{g}_{\alpha}$ with the sum extended through the positive roots outside $\langle\Theta\rangle$. It is well known that the normalizer of $\mathfrak{n}_{\Theta}^{+}$ in $\mathfrak{g}$ and $G$ are $\mathfrak{p}_{\Theta}$ and $P_{\Theta}$, respectively. Hence $\mathbb{B}_{\Theta}$ is realized also as the subset $\left\{g \cdot \mathfrak{n}_{\Theta}^{+}: g \in G\right\}$ of subalgebras conjugate to $\mathfrak{n}_{\Theta}^{+}$. We denote the maximal flag manifold $\mathbb{B}_{\emptyset}$ simply by $\mathbb{B}$.

From these standard constructions the set of flag manifolds becomes parameterized by the proper subsets of the fixed simple system of roots $\Sigma$. If $\Theta_{1} \subset \Theta_{2}$ are subsets of $\Sigma$ then $P_{\Theta_{1}} \subset P_{\Theta_{2}}$ so there exists a natural fibration $\mathbb{B}_{\Theta_{1}} \rightarrow \mathbb{B}_{\Theta_{2}}$ given by $g P_{\Theta_{1}} \rightarrow g P_{\Theta_{2}}$. The maximal flag manifold $\mathbb{B}$ fibers over all $\mathbb{B}_{\Theta}$. We denote these fibrations by $\pi$, indistinctly of the specific flag manifolds. If they are to be emphasized the projection is written $\pi_{\Theta_{2}}^{\Theta_{1}}: \mathbb{B}_{\Theta_{1}} \rightarrow \mathbb{B}_{\Theta_{2}}$.

In the sequel the notion of the flag manifold dual to $\mathbb{B}_{\Theta}$ will be required: Let $\mathcal{W}$ be the Weyl group of $G$ and denote by $w_{0} \in \mathcal{W}$ its principal involution, that is, the element of maximal length as a product of reflections with respect to the simple roots in $\Sigma$. Alternatively $w_{0}$ is the only element of $\mathcal{W}$ such that $w_{0}(\Sigma)=-\Sigma$. Put $\iota=-w_{0}$. Then $\iota(\Sigma)=\Sigma$, so that it is an involutive automorphism of the Dynkin diagram associated with $\Sigma$. For the sake of simplicity we write $\Theta^{*}=\iota(\Theta)$, if $\Theta \subset \Sigma$. The flag manifold $\mathbb{B}_{\Theta *}$ is said to be dual to $\mathbb{B}_{\Theta}$.

Put $N^{ \pm}=\exp \mathfrak{n}^{ \pm}$. The decomposition of $\mathbb{B}_{\Theta}$ into the $N^{-}$-orbits is the Bruhat decomposition of $\mathbb{B}_{\Theta}$. These orbits are given by $N^{-} w \cdot \mathfrak{p}_{\Theta}$, with $w \in \mathcal{W}$, so that its number is $\left|\mathcal{W} / \mathcal{W}_{\Theta}\right|$ where $\mathcal{W}_{\Theta}$ stands for the subgroup of $\mathcal{W}$ generated by the reflections with respect to the simple roots in $\Theta$. Just one of these orbits is open and dense in $\mathbb{B}_{\Theta}$, namely $N^{-} \cdot \mathfrak{p}_{\Theta}$. We refer to this orbit as an open (Bruhat) cell in $\mathbb{B}_{\Theta}$. This open cell has an alternative description through incidence with a nilpotent subalgebra, which will be largely used in the sequel. Let $\mathfrak{n}_{\Theta}^{-}$be the nilpotent subalgebra spanned by the root spaces complementary to $\mathfrak{p}_{\Theta}$ in $\mathfrak{g}$ :

$$
\mathfrak{n}_{\Theta}^{-}=\sum_{\alpha} \mathfrak{g}_{\alpha}
$$

with the sum extended through the negative roots outside $-\langle\Theta\rangle$. Since the Cartan involution $\theta$ takes a root $\alpha$ into $-\alpha$, it follows that $\mathfrak{n}_{\Theta}^{-}=\theta\left(\mathfrak{n}_{\Theta}^{+}\right)$. Also, $\mathfrak{n}^{-}=\theta\left(\mathfrak{n}^{+}\right)$ and $\mathfrak{n}^{+}$normalizes $\mathfrak{n}_{\Theta}^{+}$hence $\mathfrak{n}_{\Theta}^{-}$is normalized by $\mathfrak{n}^{-}$and thus by $N^{-}$.

Lemma 2.1. For a parabolic subalgebra $\mathfrak{q} \in \mathbb{B}_{\Theta}$ the following statements are equivalent:

1. $\mathfrak{q}$ belongs to the open cell $N^{-} \cdot \mathfrak{p}_{\Theta}$,

2. $\mathfrak{q} \cap \mathfrak{n}_{\Theta}^{-}=0$, and

3. $\mathfrak{n} \cap \mathfrak{n}_{\Theta}^{-}=0$ where $\mathfrak{n}$ is the nilradical of $\mathfrak{q}$.

Proof. Take $w \in \mathcal{W}$ with $w \cdot \mathfrak{p}_{\Theta} \neq \mathfrak{p}_{\Theta}$. Then $\operatorname{dim}\left(w \cdot \mathfrak{p}_{\Theta} \cap \mathfrak{n}_{\Theta}^{-}\right) \geq 1$ since $w$ interchanges root spaces, $\operatorname{dim}\left(w \cdot \mathfrak{p}_{\Theta} \cap \mathfrak{n}_{\Theta}^{-}\right) \geq 1$. Now $N^{-}$normalizes $\mathfrak{n}_{\Theta}^{-}$. Hence

$$
N^{-} \cdot\left(w \cdot \mathfrak{p}_{\Theta} \cap \mathfrak{n}_{\Theta}^{-}\right) \subset \mathfrak{n}_{\Theta}^{-}
$$


therefore any $\mathfrak{q} \in N^{-} w \cdot \mathfrak{p}_{\Theta}$ has nontrivial intersection with $\mathfrak{n}_{\Theta}^{-}$. On the other hand if $\mathfrak{q}=n \cdot \mathfrak{p}_{\Theta}$ with $n \in N^{-}$, then $\mathfrak{q} \cap \mathfrak{n}_{\Theta}^{-}=0$ for otherwise $n^{-1} \cdot\left(\mathfrak{q} \cap \mathfrak{n}_{\Theta}^{-}\right)=\mathfrak{p}_{\Theta} \cap \mathfrak{n}_{\Theta}^{-}$ would have positive dimension. This shows the equivalence between the first two statements.

The last equivalence follows the same way from the fact that $w \cdot \mathfrak{n}_{\Theta}^{+} \cap \mathfrak{n}_{\Theta}^{-} \neq 0$ if $w \cdot \mathfrak{p}_{\Theta} \neq \mathfrak{p}_{\Theta}$ (see Warner [15], Theorem 1.2.4.8).

In the sequel we say that a subset $\sigma \subset \mathbb{B}_{\Theta}$ is an open cell if $\sigma=g\left(N^{-} \cdot \mathfrak{p}_{\Theta}\right)$ for some $g \in G$. Of course any such open cell is the open orbit of a group conjugate to $N^{-}$. By the above lemma an open cell is realized as the set of parabolic subalgebras $\mathfrak{q} \in \mathbb{B}_{\Theta}$ which have null intersection with a conjugate of $\mathfrak{n}_{\Theta}^{-}$. Now we recognize the set of conjugates of $\mathfrak{n}_{\Theta}^{-}$.

Lemma 2.2. The set of subalgebras $G \cdot \mathfrak{n}_{\Theta}^{-}$identifies with the flag manifold $\mathbb{B}_{\Theta^{*}}$ dual to $\mathbb{B}_{\Theta}$.

Proof. Since $\mathfrak{n}_{\Theta}^{-}=\theta\left(\mathfrak{n}_{\Theta}^{+}\right)$this is the nilradical of the parabolic subalgebra $\theta\left(\mathfrak{p}_{\Theta}\right)$. Hence the conjugates of $\mathfrak{n}_{\Theta}^{-}$are in one-to-one correspondence with a flag manifold $\mathbb{B}_{\Theta^{\prime}}$. To see that $\Theta^{\prime}=\Theta^{*}$ observe that the restriction of $w_{0} \theta$ to $\mathfrak{a}$ is the involution $\iota$. Hence $w_{0} \theta\left(\mathfrak{p}_{\Theta}\right)=\mathfrak{p}_{\Theta^{*}}$. Therefore the set of conjugates of $\theta\left(\mathfrak{p}_{\Theta}\right)$ is $\mathbb{B}_{\Theta^{*}}$ and thus this is the flag manifold of the conjugates of $\mathfrak{n}_{\Theta}^{-}$.

Notation. The set of open Bruhat cells in $\mathbb{B}_{\Theta}$ is denoted by $\mathcal{B}_{\Theta}$ and its bijection with $\mathbb{B}_{\Theta *}$ by $x \in \mathbb{B}_{\Theta *} \mapsto \sigma_{x} \in \mathcal{B}_{\Theta}$, where $\sigma_{x}$ is the set of parabolic subalgebras in $\mathbb{B}_{\Theta}$ transversal to the nilradical of $x$. The complement of $\sigma_{x}$ is denoted by $\kappa_{x}=\mathbb{B}_{\Theta} \backslash \sigma_{x}$.

The following properties of the open cells are readily seen from the definitions.

Lemma 2.3. Take $g \in G$ and $x \in \mathbb{B}_{\Theta^{*}}$. Then $g \sigma_{x}=\sigma_{g x}$ and $g \kappa_{x}=\kappa_{g x}$. Also, any projection $\pi: \mathbb{B}_{\Theta} \rightarrow \mathbb{B}_{\Theta^{\prime}}$ is equivariant so that $\pi(\sigma) \in \mathcal{B}_{\Theta^{\prime}}$ if $\sigma$ is an open cell in $\mathbb{B}_{\Theta}$.

From Lemma 2.1] it follows that if $\mathfrak{p} \in \mathbb{B}_{\Theta}$ and $\mathfrak{q} \in \mathbb{B}_{\Theta^{*}}$, then $\mathfrak{p} \in \sigma_{\mathfrak{q}}$ if and only if $\mathfrak{n i l}(\mathfrak{p}) \cap \mathfrak{n i l}(\mathfrak{q})=0$ where $\mathfrak{n i l}(\mathfrak{p})$ stands for the nilradical of $\mathfrak{p}$. This implies the following statement at once.

Proposition 2.4. Let $x \in \mathbb{B}_{\Theta}$ and $y \in \mathbb{B}_{\Theta *}$. Then $x \in \sigma_{y}$ if and only if $y \in \sigma_{x}$.

Now we discuss the regular elements and their actions. We say that an element in $\mathfrak{g}$ (respectively in $G$ ) is split-regular if it is conjugate to some $H \in \mathfrak{a}^{+}$(respectively $h \in A^{+}=\exp \mathfrak{a}^{+}$). More generally, $X \in \mathfrak{g}$ will be said to be $\Theta$-regular if it is conjugate to $H \in \operatorname{cl}\left(\mathfrak{a}^{+}\right)$such that

$$
\Theta=\{\alpha \in \Sigma: \alpha(H)=0\} .
$$

Analogously, $g \in G$ is $\Theta$-regular if $g=\exp X$ with $X$ a $\Theta$-regular element of the Lie algebra. Of course, split-regularity and $\emptyset$-regularity are the same thing. Let $h=\exp H \in A^{+}$be $\Theta^{\prime}$-regular. Then the eigenvalues of its adjoint $\operatorname{Ad}(h)$ in $\mathfrak{n}_{\Theta}^{-}$ are $<1$ if $\Theta^{\prime} \subset \Theta$. This implies that $h^{k} \cdot \mathfrak{q} \rightarrow \mathfrak{p}_{\Theta}$, as $k \rightarrow+\infty$, for any $\mathfrak{q} \in N^{-} \cdot \mathfrak{p}_{\Theta}$. In other words, the open cell $N^{-} \cdot \mathfrak{p}_{\Theta}$ is a stable manifold for the action of $h$ in $\mathbb{B}_{\Theta}$, having $\mathfrak{p}_{\Theta}$ as attractor. The existence of such stable manifold is carried over to an arbitrary $\Theta^{\prime}$-regular element using the fact that it is conjugate to some $h \in A^{+}$:

Lemma 2.5. If $h \in G$ is $\Theta^{\prime}$-regular then for any flag manifold $\mathbb{B}_{\Theta}$, with $\Theta^{\prime} \subset \Theta$ there exists $x \in \mathbb{B}_{\Theta}$ and $\sigma \in \mathcal{B}_{\Theta}$ with $x \in \sigma$ and such that $h^{i} y \rightarrow x, i \rightarrow+\infty$, for all $y \in \sigma$. 
With the notations of this lemma we say that $x$ is the attractor of $h$ and $\sigma$ its stable manifold. In particular split-regular elements have attractors and stable manifolds in any flag manifold. We denote by $\sigma(h)$ the stable manifold of the regular element $h$.

More generally for a split-regular $h$ its set of fixed-points in the maximal flag manifold $\mathbb{B}$ is in bijection with $\mathcal{W}$. These fixed-points are the same for every element in the Weyl chamber $A^{+}$containing $h$. Hence each Weyl chamber settles a bijection of $\mathcal{W}$ with a subset of $\mathbb{B}$. The bijection is unique if we require that the identity $1 \in \mathcal{W}$ goes to the attractor of $h \in A^{+}$. In order to emphasize which Weyl chamber is under consideration we write $\mathcal{W}_{A^{+}}$for the image in $\mathbb{B}$ of this bijection, and identify it with $\mathcal{W}$. Using the bijection a fixed-point $b$ of $A^{+}$is related to some $w \in \mathcal{W}$. In this case we say that $b$ is the fixed-point of type $w$ of $h \in A^{+}$.

The following lemma shows that for any pair $(x, \sigma)$ with $x \in \sigma$ one can find a regular element having $x$ as attractor and $\sigma$ as stable manifold. It will be used frequently in the study of maximal semigroups.

Lemma 2.6. Take $\sigma \in \mathcal{B}_{\Theta}$ and $x \in \sigma$. Then there exists a $\Theta$-regular element $h \in G$ such that $x$ is its attractor and $\sigma=\sigma(h)$.

Proof. Let $b_{0}$ be the base point of $\mathbb{B}_{\Theta}=G / P_{\Theta}$ and $\sigma=N^{-} b_{0}$. If $\Theta^{\prime} \subset \Theta$ then $b_{0}$ is the attractor for any $\Theta^{\prime}$-regular element in the closure of the Weyl chamber $A^{+}$ and $\sigma$ is the stable manifold. Given $x \in \sigma$ there exists $n \in N^{-}$such that $x=n b_{0}$. So that $h_{1}=n h n^{-1}$ has $x$ as attractor and $\sigma$ as stable manifold if $h \in \operatorname{cl} A^{+}$is $\Theta^{\prime}$-regular. This shows the lemma for this specific $\sigma$. Since $G$ is transitive on $\mathcal{B}_{\Theta}$ the lemma follows by conjugation with arbitrary $g \in G$.

\section{3. $\mathcal{B}$-CONVEXITY}

Roughly speaking a subset $C$ of a flag manifold $\mathbb{B}_{\Theta}$ is said to be $\mathcal{B}$-convex provided $C$ is the intersection of all open Bruhat cells containing it. This concept of convexity is easier to develop with the aid of a convex hull operator on subsets of the flag manifolds and a duality operator * that assigns to a subset $C \subset \mathbb{B}_{\Theta}$ a subset $C^{*}$ of the dual flag manifold $\mathbb{B}_{\Theta^{*}}$. Precisely,

$$
C^{*}=\left\{x \in \mathbb{B}_{\Theta^{*}}: C \subset \sigma_{x}\right\} .
$$

Of course, this duality operator can be defined also for a subset $D \subset \mathbb{B}_{\Theta^{*}}$ giving rise to its dual $D^{*} \subset \mathbb{B}_{\Theta}$. Hence it makes sense to write $C^{* *}$, which is contained in $\mathbb{B}_{\Theta}$ if $C \subset \mathbb{B}_{\Theta}$. We put $\cos _{\mathcal{B}}(C)=C^{* *}$ and call this subset the $\mathcal{B}$-convex hull of $C$.

Accordingly $C$ is said to be $\mathcal{B}$-convex if $C=\mathrm{co}_{\mathcal{B}}(C)$.

Following Goodman and Pollack [4] (see also Goodman [3]) a convex hull operator co $(\cdot)$ deserving this name must satisfy:

1. $C \subset \operatorname{co}(C)$ for any subset $C$,

2. co $(\cdot)$ is the identity on singletons,

3 . co $(\cdot)$ is increasing with respect to inclusion of sets, and

4. co $(\cdot)$ is idempotent.

Let us discuss briefly these properties for the $\mathcal{B}$-convex hull operator. For the first one we distinguish the cases when $C^{*}$ is empty. Clearly the dual $\emptyset^{*}$ of the empty set is the whole dual flag manifold so that $\operatorname{co}_{\mathcal{B}}(C)=C^{* *}=\mathbb{B}_{\Theta}$ if $C \subset \mathbb{B}_{\Theta}$ and $C^{*}=\emptyset$. Hence $C \subset \operatorname{co}_{\mathcal{B}}(C)$ in this case. On the other hand a nonempty subset $C$ is said to be admissible if $C^{*} \neq \emptyset$, i.e., if $C \subset \sigma_{y}$ for some $y \in \mathbb{B}_{\Theta^{*}}$. For 
an admissible $C$ its $\mathcal{B}$-convex hull is seen to be the intersection of the open cells containing it. In fact, $C^{* *}=\left\{y \in \mathbb{B}_{\Theta}: C^{*} \subset \sigma_{y}\right\}$. By Proposition [2.4, $x \in \sigma_{y}$ if and only if $y \in \sigma_{x}$. Since $C^{*} \neq \emptyset$, it follows that $y \in C^{* *}$ if and only if $y \in \sigma_{x}$ for all $x \in C^{*}$. But any Bruhat cell containing $C$ is $\sigma_{x}$ for some $x \in C^{*}$. Hence for an admissible subset we have the following alternative definition.

Lemma 3.1. Suppose that $C \subset \mathbb{B}_{\Theta}$ is admissible. Then

$$
\operatorname{co}_{\mathcal{B}}(C)=\bigcap\left\{\sigma \in \mathcal{B}_{\Theta}: C \subset \sigma\right\} \text {. }
$$

Of course this implies that $C \subset \mathrm{coB}_{\mathcal{B}}(C)$. Furthermore we note that if $C$ is $\mathcal{B}$-convex then either $C=\emptyset, \mathbb{B}_{\Theta}$ or $C$ is admissible, for otherwise $\cos _{\mathcal{B}}(C)=\mathbb{B}_{\Theta}$.

Since it is irrelevant for our purposes here we do not dwell on the $\mathcal{B}$-convexity of the singletons. We just note that if $x, y \in \mathbb{B}_{\Theta}$ then there exists $\sigma \in \mathcal{B}_{\Theta}$ with $x \notin \sigma$ and $y \in \sigma$ so that $\{y\}$ is indeed $\mathcal{B}$-convex. Finally the last two of the above listed properties follow from the following statements about the duality operator:

Proposition 3.2. For a flag manifold $\mathbb{B}_{\Theta}$ the following hold:

1. If $C_{1} \subset C_{2} \subset \mathbb{B}_{\Theta}$, then $C_{1}^{*} \supset C_{2}^{*}$.

2. Let $C \subset \mathbb{B}_{\Theta}$. Then $C^{*}$ is $\mathcal{B}$-convex in $\mathbb{B}_{\Theta^{*}}$.

Proof. 1. Assuming that $C_{1} \subset C_{2}$, take $x \in C_{2}^{*}$. Then $C_{2} \subset \sigma_{x}$ so that $C_{1} \subset \sigma_{x}$. This implies that $x \in C_{1}^{*}$.

2. If $C$ is not admissible then $C^{*}=\emptyset, \mathbb{B}_{\Theta^{*}}$ and its $\mathcal{B}$-convexity is trivial. Assuming that $C^{*} \neq \emptyset$ we must check that $C^{*}=\left(C^{*}\right)^{* *}$. The inclusion $C^{*} \subset\left(C^{*}\right)^{* *}$ is equivalent to $C^{*} \subset \operatorname{co}_{\mathcal{B}}\left(C^{*}\right)$, showed above. On the other hand take $y \in\left(C^{*}\right)^{* *}$. Then $x \in \sigma_{y}$ for every $x \in C^{* *}$. In particular $x \in \sigma_{y}$ for all $x \in C$ because $C$ is contained in $C^{* *}$. By Proposition 2.4, $y \in \sigma_{x}$, implying that $y \in C^{*}$, showing that $\left(C^{*}\right)^{* *} \subset C^{*}$.

From this proposition we easily get the following properties of the operator $\cos _{\mathcal{B}}(\cdot)$ :

Proposition 3.3. For a flag manifold $\mathbb{B}_{\Theta}$ the following hold:

1. If $C_{1} \subset C_{2}$, then $\cos _{\mathcal{B}}\left(C_{1}\right) \subset \operatorname{cop}_{\mathcal{B}}\left(C_{2}\right)$.

2. If $C \subset \mathbb{B}_{\Theta}$, then $\operatorname{co}_{\mathcal{B}}(C)=\cos _{\mathcal{B}}\left(\cos _{\mathcal{B}}(C)\right)$.

Proof. The first property is a direct consequence of the previous proposition. Also by the proposition $\mathrm{co}_{\mathcal{B}}(C)$ is $\mathcal{B}$-convex, so that $\operatorname{co}_{\mathcal{B}}(C)=\operatorname{co}_{\mathcal{B}}\left(\operatorname{co}_{\mathcal{B}}(C)\right)$.

3.1. Examples. The examples below illustrate that the $\mathcal{B}$-convex sets may be either arbitrary sets or sets which resemble the standard convex sets in affine spaces or in Riemannian manifolds.

1. In case $\mathfrak{g}$ is a Lie algebra with real rank one there exists just one flag manifold $\mathbb{B}$ which is diffeomorphic to a sphere in some dimension. The Bruhat decomposition of $\mathbb{B}$ has two components - the open one and its complement which is a singleton. Thus $\mathcal{B}$ consists of the subsets $\mathbb{B} \backslash\{x\}, x \in \mathbb{B}$. Therefore any subset of $\mathbb{B}$ is $\mathcal{B}$-convex.

2. Let $\mathfrak{g}=\mathfrak{s l}(n, \mathbb{R})$. The flag manifolds are the standard manifolds of flags of subspaces in $\mathbb{R}^{n}$. In particular the Grassmannians, including the projective space, are flag manifolds of Lie groups associated with $\mathfrak{s l}(n, \mathbb{R})$. Let us focus attention on the Grassmannian $\operatorname{Gr}_{k}(n)$ of $k$-dimensional subspaces of $\mathbb{R}^{n}$. A direct check of the isotropy subalgebras of the $\operatorname{Sl}(n, \mathbb{R})$-action on 
$\operatorname{Gr}_{k}(n)$ shows that its dual is the Grassmannian $\mathrm{Gr}_{n-k}(n)$ of subspaces having complementary dimension. In more concrete terms this duality is given by incidence between $k$-dimensional and $(n-k)$-dimensional subspaces of $\mathbb{R}^{n}$. Indeed an open cell is the stable manifold of the attractor for the action of a split regular element $h$ in the group. In the present case $h$ is a diagonalizable matrix in $\mathrm{Sl}(n, \mathbb{R})$ having positive and distinct eigenvalues. If $\left\{e_{1}, \ldots, e_{n}\right\}$ is a basis of eigenvectors of $h$, then the subspace spanned by $\left\{e_{1}, \ldots, e_{k}\right\}$ is the attractor of $h$ in $\operatorname{Gr}_{k}(n)$. Its stable manifold is easily seen to be the open and dense subset of $k$-dimensional subspaces transversal to $\operatorname{span}\left\{e_{k+1}, \ldots, e_{n}\right\}$. This implies that for each $U \in \mathrm{Gr}_{n-k}(n)$ its associated open cell is

$$
\sigma_{U}=\left\{V \in \operatorname{Gr}_{k}(n): V \cap U=0\right\},
$$

while $\kappa_{U}$ is the set of $k$-dimensional subspaces meeting $U$ nontrivially. It follows that $\emptyset \neq C \subset \operatorname{Gr}_{k}(n)$ is admissible if and only if there exists a $(n-k)$-dimensional subspace $U$ such that $V \cap U=0$ for all $V \in C$. Note that as in the case of rank one groups there are rather arbitrary $\mathcal{B}$-convex subsets in the Grassmannians. In fact, for any admissible $D \subset \mathrm{Gr}_{n-k}(n)$, its dual $D^{*}$ is $\mathcal{B}$-convex in $\operatorname{Gr}_{k}(n)$.

For $k=1$ we can single out a nice class of $\mathcal{B}$-convex sets, namely the classical convex subsets in the projective space $\mathbb{P}^{n-1}$ : Let $W \subset \mathbb{R}^{n}$ be a pointed convex cone and denote by $[W]$ the set of lines in $\mathbb{P}^{n-1}$ contained in $W$. Since $W$ is pointed $[W]$ is admissible. Also, $W$ is the intersection of the half-spaces in $\mathbb{R}^{n}$ containing it. Hence $[W]$ is $\mathcal{B}$-convex in $\mathbb{P}^{n-1}$. Of course, not every $\mathcal{B}$-convex set is constructed this way from a convex cone.

3. We continue with $\mathfrak{g}=\mathfrak{s l}(n, \mathbb{R})$. Let $\mathbf{r}=\left(r_{1}<\cdots<r_{m}\right)$ be a sequence of integers with $1 \leq r_{1}$ and $r_{m} \leq n-1$ and denote by $\mathbb{F}(\mathbf{r})$ the manifold of flags

$$
\left(V_{1} \subset \cdots \subset V_{m}\right)
$$

of subspaces of $\mathbb{R}^{n}$ with $\operatorname{dim} V_{i}=r_{i}$. Put $\overline{\mathbf{r}}=\left(n-r_{m}<\cdots<n-r_{1}\right)$. Then $\mathbb{F}(\overline{\mathbf{r}})$ is the flag manifold dual to $\mathbb{F}(\mathbf{r})$. As in the Grassmannian case this can be seen either by looking at the isotropy subalgebras or by verifying directly that the open cells are given by incidence between the subspaces in a flag. Indeed, if $U=\left(U_{1} \subset \cdots \subset U_{m}\right) \in \mathbb{F}(\overline{\mathbf{r}})$, then

$$
\sigma_{U}=\left\{\left(V_{1} \subset \cdots \subset V_{m}\right): V_{i} \cap U_{m-i+1}=0, i=1, \ldots, m\right\}
$$

is an open cell in $\mathbb{F}(\mathbf{r})$.

3.2. Topology. Up to this point we have considered $\mathcal{B}$-convexity for arbitrary subsets of the flag manifolds, looking at the incidence of parabolic subalgebras only. Now we consider some topological properties of the duality and $\mathcal{B}$-convex hull operators.

Since a flag manifold $\mathbb{B}_{\Theta}$ is a homogenous space of $G$, it is endowed with the quotient topology, rendering it a compact metrizable space. This topology is given also by embedding $\mathbb{B}_{\Theta}$ in a Grassmannian, either by identifying it with the subalgebras conjugate to $\mathfrak{p}_{\Theta}$ or to $\mathfrak{n}_{\Theta}^{+}$. Here the topology in a Grassmannian is the standard one. A basic property of this topology is: Let $L$ be a vector space with $\operatorname{dim} L=n$. Denote by $\operatorname{Gr}_{k}(L)$ the Grassmannian of $k$-dimensional subspaces of $L$. Suppose that $\xi_{0} \in \operatorname{Gr}_{k}(L)$ and $\eta_{0} \in \mathrm{Gr}_{n-k}(L)$ are transversal, i.e., $\xi_{0} \cap \eta_{0}=0$. Then there are neighborhoods $A \ni \xi_{0}$ and $B \ni \eta_{0}$ in $\mathrm{Gr}_{k}(L)$ and $\mathrm{Gr}_{n-k}(L)$, respectively, such that $\xi \cap \eta=0$ for all $\xi \in A$ and $\eta \in B$. 
Now recall that an open cell $\sigma_{\mathfrak{q}}, \mathfrak{q} \in \mathbb{B}_{\Theta^{*}}$, is the set of parabolic subalgebras in $\mathbb{B}_{\Theta}$ which are transversal to the nilradical $\mathfrak{n i l}(\mathfrak{q})$ of $\mathfrak{q}$. Since the dimension of $\mathfrak{n i l}(\mathfrak{q})$ complements the dimension of any $\mathfrak{p} \in \mathbb{B}_{\Theta}$, the above transversality property implies the

Lemma 3.4. Let $x_{0} \in \mathbb{B}_{\Theta}$ and $y_{0} \in \mathbb{B}_{\Theta^{*}}$ be such that $y_{0} \in \sigma_{x_{0}}$ (and hence $x_{0} \in$ $\left.\sigma_{y_{0}}\right)$. Then there are neighborhoods $U \ni x_{0}$ and $V \ni y_{0}$ in $\mathbb{B}_{\Theta}$ and $\mathbb{B}_{\Theta *}$ respectively such that $x \in \sigma_{y}$ (and hence $y \in \sigma_{x}$ ), for all $x \in U$ and $y \in V$.

Another basic property of the topology in the flag manifolds is related to sequences in the complements $\kappa_{y}$ of the open cells $\sigma_{y}$ :

Lemma 3.5. Let $y_{j} \in \mathbb{B}_{\Theta^{*}}$ be a sequence with $\lim y_{j}=y$. If $x \in \kappa_{y}$ then there exists a sequence $x_{j} \in \kappa_{y_{j}}$ such that $\lim x_{j}=x$.

Proof. By transitivity of $G$ in $\mathbb{B}_{\Theta *}$ there exists a sequence $g_{j} \in G$ with $g_{j} \rightarrow 1$ and such that $y_{j}=g_{j} y$. The required sequence is $x_{j}=g_{j} x$. In fact, $x_{j} \in \kappa_{y_{j}}=g_{j} \kappa_{y}$ and $x_{j} \rightarrow x$.

From these lemmas we get the following topological properties of the duality operator which are required in the study of maximal semigroups.

Proposition 3.6. Suppose that $C \subset \mathbb{B}_{\Theta}$ is compact and admissible. Then $C^{*}$ is open.

Proof. Suppose that $C \neq \emptyset \neq C^{*}$ and take $x \in C$ and $y \in C^{*}$. By Lemma 3.4 above there are neighborhoods $U_{x} \ni x$ and $V_{x} \ni y$ such that $z \in \sigma_{w}$ for all $z \in U_{x}$ and $w \in V_{x}$. By compactness there exists a finite covering

$$
C \subset U_{x_{1}} \cup \cdots \cup U_{x_{l}}
$$

Then $V=V_{x_{1}} \cap \cdots \cap V_{x_{l}}$ is a neighborhood of $y$ contained in $C^{*}$.

Proposition 3.7. Let $C \subset \mathbb{B}_{\Theta}$ be admissible with int $C \neq \emptyset$. Then $\operatorname{cl}\left(C^{*}\right) \subset \sigma_{x}$ for all $x \in \operatorname{int} C$. Hence $\operatorname{cl}\left(C^{*}\right) \subset(\operatorname{int} C)^{*}$ and $\operatorname{cl}\left(C^{*}\right)$ is admissible.

Proof. Take $x \in \operatorname{int} C$. Let $y \in \operatorname{cl}\left(C^{*}\right)$ and $y_{j} \in C^{*}$ be such that $\lim y_{j}=y$. We must check that $x \in \sigma_{y}$. Suppose to the contrary that $x \in \kappa_{y}$. Then by Lemma 3.5 there exists a sequence $x_{j} \in \kappa_{y_{j}}$ with $\lim x_{j}=x$. This implies that $x_{j} \in \operatorname{int} C$ for large $j$. But this contradicts the fact that $y_{j} \in C^{*} \subset(\operatorname{int} C)^{*}$.

Proposition 3.8. Let $C \subset \mathbb{B}_{\Theta}$ be open and such that $\mathrm{cl} C$ is admissible. Then $C^{*}$ is closed and $\operatorname{int}\left(C^{*}\right)=(\mathrm{cl} C)^{*}$.

Proof. Since $C$ is open, Proposition 3.7 implies that $\operatorname{cl}\left(C^{*}\right) \subset C^{*}$ so that $C^{*}$ is closed. Furthermore, by Proposition [3.2 $(\mathrm{clC})^{*} \subset C^{*}$. But $(\mathrm{cl} C)^{*}$ is open hence $(\mathrm{clC})^{*} \subset \operatorname{int}\left(C^{*}\right)$. For the reverse inclusion suppose that there exists $x \in$ int $\left(C^{*}\right) \backslash(\mathrm{cl} C)^{*}$. Then $x \in \kappa_{y}$ for some $y \in \operatorname{cl} C$. Take a sequence $y_{j} \in C$ such that $\lim y_{j}=y$. By Lemma [3.5 there exists a sequence $x_{j} \in \kappa_{y_{j}}$ with $\lim x_{j}=x$. Hence, for large $j, x_{j} \in \operatorname{int}\left(C^{*}\right) \subset C^{*}$ and $x_{j} \in \kappa_{y_{j}}$ with $y_{j} \in C$, which is a contradiction.

Applying this proposition twice we get the following information about the $\mathcal{B}$ convex hull of a closed subset. 
Proposition 3.9. Let $C \subset \mathbb{B}_{\Theta}$ be a closed admissible subset with $\operatorname{int} C \neq \emptyset$. Then $\mathrm{co}_{\mathcal{B}}(C)$ is closed and has nonempty interior

$$
\operatorname{int}\left(\operatorname{co}_{\mathcal{B}}(C)\right)=\left(\operatorname{cl}\left(C^{*}\right)\right)^{*} .
$$

Proof. By Proposition 3.6, $C^{*}$ is open hence $\operatorname{co}_{\mathcal{B}}(C)=C^{* *}$ is closed. The above proposition applied to $C^{*}$ implies that

$$
\operatorname{int}\left(C^{* *}\right)=\left(\operatorname{cl}\left(C^{*}\right)\right)^{*} .
$$

This open set is not empty because $\mathrm{cl}\left(C^{*}\right)$ is admissible by Proposition 3.7

3.3. Invariance. The relevance of $\mathcal{B}$-convexity for semigroups in $G$ stays in the following invariance properties of the dual and the $\mathcal{B}$-convex hull operators.

Proposition 3.10. Let $g \in G$ and $C \subset \mathbb{B}_{\Theta}$. Then $(g C)^{*}=g\left(C^{*}\right)$.

Proof. Take a parabolic subalgebra $\mathfrak{p} \in C^{*}$ and denote its nilradical by $\mathfrak{n}$. By definition $\mathfrak{q} \cap \mathfrak{n}=0$ for every parabolic subalgebra $\mathfrak{q} \in C$. Now $g \cdot \mathfrak{n}$ is the nilradical of $g \cdot \mathfrak{p}$, and

$$
g \cdot \mathfrak{q} \cap g \cdot \mathfrak{n}=g \cdot(\mathfrak{q} \cap \mathfrak{n})=0
$$

if $\mathfrak{q} \in C$. This implies that $g \cdot \mathfrak{p} \in(g C)^{*}$ and hence that $g\left(C^{*}\right) \subset(g C)^{*}$. Applying this inclusion to $g C$ and $g^{-1}$ we have $g^{-1}\left((g C)^{*}\right) \subset C^{*}$ so that $(g C)^{*} \subset g\left(C^{*}\right)$, concluding the proof.

Corollary 3.11. Let $g \in G$ and $C \subset \mathbb{B}_{\Theta}$ be such that $g C \subset C$. Then $g^{-1}\left(C^{*}\right) \subset$ $C^{*}$.

Proof. Proposition 3.3 ensures that $(g C)^{*} \supset C^{*}$. Hence by the above proposition $g\left(C^{*}\right) \supset C^{*}$ which is equivalent to $g^{-1}\left(C^{*}\right) \subset C^{*}$.

Corollary 3.12. Let $g \in G$ and $C \subset \mathbb{B}_{\Theta}$. Then $g\left(\operatorname{co}_{\mathcal{B}}(C)\right)=\operatorname{co}_{\mathcal{B}}(g C)$. Therefore $g C$ is $\mathcal{B}$-convex if $C$ is $\mathcal{B}$-convex.

Proof. Follows from the proposition and the equality $\operatorname{co}_{\mathcal{B}}(C)=C^{* *}$.

Now we can prove that the $\mathcal{B}$-convex hull operator maps invariant subsets into invariant subsets. This will be used in the description of maximal semigroups.

Proposition 3.13. Suppose that $g \in G$ and $C \subset \mathbb{B}_{\Theta}$ are such that $g C \subset C$. Then $g\left(\operatorname{co}_{\mathcal{B}}(C)\right) \subset \operatorname{co}_{\mathcal{B}}(C)$.

Proof. Follows immediately from the previous corollary and Proposition 3.3.

As a consequence of these facts we get the following localization type property of the $\mathcal{B}$-convex sets:

Proposition 3.14. The family of open $\mathcal{B}$-convex sets is a basis for the topology of $\mathbb{B}_{\Theta}$.

Proof. Let $C \subset \mathbb{B}_{\Theta^{*}}$ be a compact admissible subset with $\operatorname{int} C \neq \emptyset$. From the previous subsection we know that $C^{*}$ is open and its closure is admissible. Clearly $C^{*}$ is an open $\mathcal{B}$-convex set. From it we generate a basis for the open sets in $\mathbb{B}_{\Theta}$. First take $x \in C^{*}$ and an open cell $\sigma \supset \mathrm{cl}^{*}$. By Lemma [2.6] there exists a splitregular $h \in G$ such that $x$ is its attractor and $\sigma=\sigma(h)$. The sequence $h^{k}$ contracts $\sigma$ into $x$ as $k \rightarrow+\infty$. Since $\operatorname{cl}\left(C^{*}\right)$ is a compact subset of $\sigma$, the contraction is uniform in $\operatorname{cl}\left(C^{*}\right)$. This means that for any neighborhood $U$ of $x$ there exists $k_{0}>0$ 
such that $h^{k} C^{*} \subset U$ for $k \geq k_{0}$. Therefore the open $\mathcal{B}$-convex sets form a basis for the neighborhoods of $x$. The proposition follows then by transitivity of $G$ and the fact that $g \in G$ maps $\mathcal{B}$-convex sets into $\mathcal{B}$-convex sets.

Remark 3.15. It was observed by D. Mittenhuber in personal communication that the above results about $\mathcal{B}$-convex are fully extended to a general pair of relations $R_{1} \subset X \times Y$ and $R_{2} \subset Y \times X$, relating the sets $X$ and $Y$, as far as the relations satisfy the symmetric property $x R_{1} y$ if and only if $y R_{2} x$.

\section{Semigroups}

In this section we consider the action on the flag manifolds of semigroups in semi-simple Lie groups. We complement the results of San Martin [12] and San Martin and Tonelli [14, paving the way for the characterization of the maximal semigroups.

4.1. Topological introduction. Before looking at the semigroup actions on the flag manifolds we recall some terminology of a topological nature which hold in a more general context. In this subsection we let $G$ be a topological group acting continuously in a topological space $M$. Let $S \subset G$ be a semigroup with $\operatorname{int} S \neq \emptyset$.

Its action on $M$ induces the pre-order relation $x \preceq y$ if $y \in \operatorname{cl}(S x), x, y \in M$. Let $\sim$ be the equivalence relation associated with $\preceq$, namely $x \sim y$ if $x \preceq y$ and $y \preceq x$. An equivalence class $D$ of $\sim$ satisfies $D \subset \operatorname{cl}(S x)$ for all $x \in D$, and is maximal with this property. The pre-order in $M$ induces a partial order in the quotient $M / \sim$ which is also denoted by $\preceq$.

A control set for $S$ in $M$ is an equivalence class $D$ of $\sim$ having the property that there exists $x \in D$ and $g \in \operatorname{int} S$ with $g x=x$. We note that this definition of control set amounts to the effective control sets of 14. Given a control set $D$ the fixed-point set

$$
D_{0}=\{x \in D: \exists g \in \operatorname{int} S, g x=x\}
$$

is known to be open and dense in $D$. It is called the core or set of transitivity of $D$ (see [14). This second name comes from the fact that for all $x, y \in D_{0}$ there exists $g \in S$ such that $g x=y$. We denote by $\mathcal{D}(S)$ the set of control sets of $S$. It is partially ordered by $\preceq$ in $M / \sim$. In case $M$ is compact there are invariant control sets. These are control sets which are maximal with respect to $\preceq$. They are closed subsets of $M$. The same way there are minimal control sets, which are open and coincide with the cores of the invariant control sets of the inverse semigroup $S^{-1}=\left\{g^{-1}: g \in S\right\}$.

The domain of attraction $\mathcal{A}(D)$ of a control set $D$ is defined by

$$
\mathcal{A}(D)=\{x \in M: \exists g \in S, g x \in D\} .
$$

For a subset $C$ contained in $M$ we denote by $S_{C}$ its compression semigroup in $G$ :

$$
S_{C}=\{g \in G: g C \subset C\} .
$$

It follows immediately that $S_{C}=S_{\operatorname{int}(C)}$ if $C=\operatorname{cl}(\operatorname{int} C)$. We refer to Colonius and Kliemann [2] for a detailed development of these concepts in the context of control systems. 
4.2. Flag manifolds. We return here to the flag manifold setting with $S$ a semigroup with nonvoid interior in the semi-simple group $G$.

Consider for a moment the maximal flag manifold $\mathbb{B}=\mathbb{B}_{\emptyset}$. From 14 we know that for each $w \in \mathcal{W}$ there exists a control set $D(w)$ such that $x \in D(w)_{0}$ if and only if $x$ is the fixed point of type $w$ for some split-regular $h \in \operatorname{int} S$. Moreover, any control set $D$ is $D(w)$ for some $w \in \mathcal{W}$. The assignment $w \mapsto D(w)$ permits us to single out, from $S$, a flag manifold $\mathbb{B}(S)$ as follows: Take a split-regular $h \in \operatorname{int} S$ and denote by $A^{+}=\exp \mathfrak{a}^{+}$the Weyl chamber containing $h$. Recall that we write $\mathcal{W}_{A^{+}}$to the set of $A^{+}$-fixed-points in $\mathbb{B}$, and identify it with $\mathcal{W}$. Let $1 \in \mathcal{W}_{A^{+}}$be the identity. Then the control set $D(1)$ is the only invariant control set in $\mathbb{B}$. The same way the control set $D\left(w_{0}\right)$ is the only minimal control set in $\mathbb{B}$ where $w_{0}$ is the principal involution of $\mathcal{W}$.

The subset $\mathcal{W}_{A^{+}}(S)=\{w \in W: D(w)=D(1)\}$ is a parabolic subgroup of $\mathcal{W}_{A^{+}}$, that is, it is generated by the reflections with respect to the simple roots in a proper subset $\Theta(S) \subset \Sigma$. Here $\Sigma$ is the simple system of roots associated with $\mathfrak{a}^{+}$. We put $\mathbb{B}(S)=\mathbb{B}_{\Theta(S)}$. A decisive property of this special flag manifold is that the invariant control set of $S$ on it is an admissible subset, i.e., is contained in open Bruhat cells. Precisely,

Proposition 4.1. With the above notations let $C \subset \mathbb{B}(S)$ be the invariant control set. Then $C$ is contained in the stable manifold $\sigma(h)$ for any split-regular $h \in \operatorname{int} S$. Moreover if $\Theta \subset \Theta(S)$ and $\pi: \mathbb{B}_{\Theta} \rightarrow \mathbb{B}(S)$ is the canonical fibration, then $\pi^{-1}(C)$ is the invariant control set for $S$ in $\mathbb{B}_{\Theta}$.

Proof. See Proposition 4.8 and Theorem 4.3 in 14].

In the sequel we say that the semigroup is of parabolic type $\Theta$ if $\Theta(S)=\Theta$, i.e., $\mathbb{B}(S)=\mathbb{B}_{\Theta}$. We emphasize that any proper semigroup with nonempty interior is of parabolic type $\Theta$ for some $\Theta$. Furthermore if $S \subset T$ are semigroups with nonempty interior, then any control set of $S$ is contained in just one control set of $T$, and $T$ is of parabolic type $\Theta^{\prime} \supset \Theta$ if $S$ is of parabolic type $\Theta$.

Another piece of information provided by the subgroup $\mathcal{W}_{A^{+}}(S)$ concerns the number of control sets in the flag manifold $\mathbb{B}_{\Theta}$. It is given by the order of the double coset space $\mathcal{W}_{A^{+}}(S) \backslash \mathcal{W} / \mathcal{W}_{\Theta}$, where $\mathcal{W}_{\Theta}$ is the parabolic subgroup generated by the reflections in $\Theta$.

For a semigroup of parabolic type $\Theta$ its invariant control set in $\mathbb{B}_{\Theta}$ is an admissible subset which is the closure of its interior. The next proposition complements this statement by showing that every subset of $\mathbb{B}_{\Theta}$ having these properties is the invariant control set of some semigroup of parabolic type $\Theta$.

Proposition 4.2. Suppose that $C \subset \mathbb{B}_{\Theta}$ is admissible and satisfies $C=\operatorname{cl}(\operatorname{int} C)$. Then the compression semigroup

$$
S_{C}=\{g \in G: g C \subset C\}
$$

has nonempty interior. Moreover $C$ is the invariant control set of $S_{C}$ in $\mathbb{B}_{\Theta}, C_{0}=$ int $C$ and the parabolic type of $S_{C}$ is $\Theta$.

Proof. Take $x \in \operatorname{int} C$ and let $\sigma$ be an open cell containing $C$. By Lemma 2.6 there exists a split-regular $h \in G$ such that $x$ is its attractor and $\sigma=\sigma(h)$. The sequence $h^{k}$ contracts $\sigma$ into $x$ as $k \rightarrow+\infty$. Since $C$ is a compact subset of $\sigma$, the contraction is uniform in $C$. This means that for any neighborhood $U$ of $x$ there exists $k_{0}>0$ such that $h^{k} C \subset U$ for $k \geq k_{0}$. In particular if we take $U \subset C$ we 
find that $g=h^{k_{0}}$ belongs to $S_{C}$. Furthermore the subset $\{f: f(C) \subset U\}$ is open in the compact-open topology on the continuous maps of $\mathbb{B}_{\Theta}$. By the continuity of the $G$-action we have then that $g \in \operatorname{int} S_{C}$ showing the first part of the proposition.

For the second statement note that $C$ is invariant under $S_{C}$. Moreover, we found a split-regular $g \in \operatorname{int} S$ having $x$ as the attractor for arbitrary $x \in \operatorname{int} C$. This implies that $C$ is the invariant control set of $S_{C}$ because attractors for the split-regular elements in $\operatorname{int} S$ are contained in the core of the invariant control set.

\section{Maximal Semigroups}

A subsemigroup $S$ of a group $L$ is said to be maximal if it is not a group and is not contained properly in any semigroup $T \subset L, T \neq L$. It is well known that any proper semigroup with interior points is contained in a maximal semigroup, which is by force closed. See Hilgert, Hofmann and Lawson [5] for a proof of this fact using the Lemma of Zorn.

For semigroups with nonempty interior in semi-simple Lie groups we can enlarge the notion of maximality by taking into account the parabolic type of the semigroup. As before let $G$ be a semi-simple Lie group.

Definition 5.1. We say that a semigroup $S \subset G$ with $\operatorname{int} S \neq \emptyset$ is $\Theta$-maximal or maximal with respect to $\mathbb{B}_{\Theta}$ if its parabolic type is $\Theta$ and is not properly contained in any semigroup of parabolic type $\Theta$.

It will be proved below that the $\Theta$-maximal semigroups are essentially the compression semigroups of the $\mathcal{B}$-convex sets in $\mathbb{B}_{\Theta}$. Before providing the proof for this fact we make the following remarks:

Let $S$ be a $\Theta$-maximal semigroup and denote by $C$ its invariant control set in $\mathbb{B}_{\Theta}$. Since $C$ is $S$-invariant it follows that $S \subset S_{C}$ where $S_{C}$ is the compression semigroup

$$
S_{C}=\{g \in G: g C \subset C\} .
$$

By Proposition 4.2 $S_{C}$ is of parabolic type $\Theta$. Therefore $S$ is the compression semigroup of its invariant control set in $\mathbb{B}_{\Theta}$ if it is $\Theta$-maximal. Suppose there exists $\Theta^{\prime} \neq \Sigma$ containing $\Theta$ properly and let $\pi: \mathbb{B}_{\Theta} \rightarrow \mathbb{B}_{\Theta^{\prime}}$. Then $\pi(C)$ is admissible in $\mathbb{B}_{\Theta^{\prime}}$. Moreover int $(\pi(C))$ is dense in $\pi(C)$ because $\pi$ is an open map. Hence $S_{\pi(C)}$ is of parabolic type $\Theta^{\prime}$ by Proposition 4.2. Since $\pi$ is equivariant under the actions of $G$ in $\mathbb{B}_{\Theta}$ and $\mathbb{B}_{\Theta^{\prime}}$ it follows that $S \subset S_{\pi(C)}$. This inclusion is proper. In fact, the invariant control set of $S_{\pi(C)}$ in $\mathbb{B}_{\Theta}$ is $\pi^{-1}(\pi(C))$ because $S_{\pi(C)}$ is of parabolic type $\Theta^{\prime}$. But $C$ is admissible in $\mathbb{B}_{\Theta}$, so that no fiber of $\pi$ is contained in $C$. This implies that $C \neq \pi^{-1}(\pi(C))$. Hence the two semigroups have different invariant control sets. Therefore $S \neq S_{\pi(C)}$. From this we deduce that any semigroup of parabolic type $\Theta$ is contained properly in a semigroup of parabolic type $\Theta^{\prime} \supset \Theta$ if $\Theta \neq \Theta^{\prime}$. In particular a $\Theta$-maximal semigroup is not maximal unless $\Theta$ is maximal in $\Sigma$, that is, the complement of a singleton. In this case $\mathbb{B}_{\Theta}$ is a minimal flag manifold.

Conversely, if $\Theta$ is maximal and $S$ is a $\Theta$-maximal semigroup then $S$ is maximal. In fact, if $T \subset S$ and $T \neq S$ then $T$ is of parabolic type $\Theta^{\prime} \supset \Theta$. Since $S$ is $\Theta$-maximal this implies that $\Theta^{\prime} \neq \Theta$, but then $T$ cannot be a proper semigroup.

Now, thanks to the invariance of the $\mathcal{B}$-convex hull of a subset it follows easily that a $\Theta$-maximal semigroup is the compression semigroup of a $\mathcal{B}$-convex set in $\mathbb{B}_{\Theta}$ : 
Proposition 5.2. Suppose that $S$ is a $\Theta$-maximal semigroup and denote by $C$ its invariant control set in $\mathbb{B}_{\Theta}$. Put $K=\operatorname{cl}\left(\operatorname{int}\left(\cos _{\mathcal{B}}(C)\right)\right)$. Then $C=K$ and

$$
S=S_{C}=\{g \in G: g C \subset C\} .
$$

Proof. If $g \in S$ then $g C \subset C$ so that Proposition 3.13 ensures that $g\left(\cos _{\mathcal{B}}(C)\right) \subset$ $\operatorname{co}_{\mathcal{B}}(C)$. By continuity $g K \subset K$. Hence $S$ is contained in the compression semigroup $S_{K}$ of $K$. By definition of a semigroup of parabolic type $\Theta, C$ is admissible in $\mathbb{B}_{\Theta}$. This implies that $K$ is contained in an open cell $\sigma$ of $\mathbb{B}_{\Theta}$. It follows that $K$ is a nonempty admissible subset satisfying $K=\mathrm{cl}(\operatorname{int} K)$. Therefore Proposition 4.2 implies that $S_{K}$ is of parabolic type $\Theta$. Now by assumption $S$ is $\Theta$-maximal. Hence $S=S_{K}$. Invoking Proposition 4.2 again we have that the invariant control set of $S_{K}$ is $K$ so that $C=K$ concluding the proof.

This proposition has the following converse which ensures that the compression semigroup of the interior of a $\mathcal{B}$-convex set is maximal.

Proposition 5.3. Let $C \subset \mathbb{B}_{\Theta}$ be a proper closed $\mathcal{B}$-convex set with $\operatorname{int} C \neq \emptyset$. Put $K=\operatorname{cl}(\operatorname{int} C)$. Then the compression semigroup $S_{K}$ is $\Theta$-maximal.

Proof. By definition of $\mathcal{B}$-convexity $C$ is admissible. Then Proposition 3.9 implies that $K$ is admissible. Since $K=\operatorname{cl}(\operatorname{int} K)$ it follows from Proposition 4.2 that $\operatorname{int} S_{K} \neq \emptyset, S_{K}$ is of parabolic type $\Theta$ and $K$ is the invariant control set of $S_{K}$. To see the $\Theta$-maximality take a semigroup $T$ of parabolic type $\Theta$ with $S_{K} \subset T$. Denote by $D$ the invariant control set of $T$ in $\mathbb{B}_{\Theta}$. From $S_{K} \subset T$ it follows that $K \subset D$. Now, $S_{K}$ is a compression semigroup and $D$ is $T$-invariant. Hence it is enough to show that $K=D$ to get $T \subset S_{K}$ and thus $S_{K}=T$.

We prove first that $D \subset \operatorname{co}_{\mathcal{B}}(K)$. Suppose to the contrary that there exists $y \in D \backslash \operatorname{co}_{\mathcal{B}}(K)$. By definition of $\mathcal{B}$-convexity there exists an open cell $\sigma \in \mathcal{B}_{\Theta}$ such that $K \subset \sigma$ and $y \notin \sigma$. Take $x \in \operatorname{int} K$. By Lemma 2.6 there exists a splitregular $h \in G$ such that $x$ is its attractor and $\sigma=\sigma(h)$. Arguing as in the proof of Proposition 4.2 we can assume, after replacing $h$ by some of its powers $h^{p}, p \geq 1$, that $h \in \operatorname{int} S_{K}$.

The limit $y_{0}=\lim _{j \rightarrow+\infty} h^{j} y$ is a fixed point of $h$ different from the attractor $x$ because $y \notin \sigma(h)$. Since $h \in \operatorname{int} S_{K}$ there exists a control set, say $E$, of $S_{K}$ such that $y_{0} \in E_{0}$. The fact that $y_{0}$ is not the attractor of $h$ implies that $E \neq K$. On the other hand $h \in T, y \in D$ and $D$ is closed and $T$-invariant. Hence $y_{0} \in D$. But $E$ is entirely contained in a control set of $T$. Therefore $E \subset D$.

Now, both $S_{K}$ and $T$ are of parabolic type $\Theta$ so that they have the same number of control sets in $\mathbb{B}_{\Theta}$. Since any control set of $S_{K}$ is contained in a control set of $T$, the existence of $E \neq K$ with $K, E \subset D$ is a contradiction. This shows that $D \subset \operatorname{co} \mathcal{B}(K)$.

Therefore $\operatorname{int} D \subset \operatorname{int}\left(\operatorname{co}_{\mathcal{B}}(K)\right)$. But int $\left(\operatorname{co}_{\mathcal{B}}(K)\right)=\operatorname{int} C$ by Proposition [3.9] On the other hand $D=\operatorname{cl}(\operatorname{int} D)$ because it is the invariant control set of a semigroup with nonvoid interior. Hence $D \subset \operatorname{cl}(\operatorname{int} C)=K$. This implies that $T=S_{K}$, showing that $S_{K}$ is $\Theta$-maximal.

We summarize the previous propositions in the following final characterization of maximal semigroups in semi-simple Lie groups.

Theorem 5.4. A semigroup $S$ is $\Theta$-maximal if and only if there exists a $\mathcal{B}$-convex set $C$ with int $C \neq \emptyset$ such that $S=S_{K}$, the compression semigroup of $K=\operatorname{cl}(\operatorname{int} C)$. In this case $K$ is the invariant control set of $S$ in $\mathbb{B}_{\Theta}$ and $\operatorname{co}_{\mathcal{B}}(K) \subset C$. 
A semigroup $S$ is maximal if and only if $\mathbb{B}_{\Theta}$ is a minimal flag manifold and $S$ is $\Theta$-maximal.

\section{Miscellanea}

In this section we prove further results about maximal semigroups and provide some examples.

6.1. Duality and minimal control set. Since a $\Theta$-maximal semigroup $S$ is the compression semigroup of its invariant control set $C$ every object related to $S$ is in principle obtained from $C$. We determine here the minimal control set using the duality operator. This control set is the core of the invariant control set of $S^{-1}=\left\{g^{-1}: g \in S\right\}$ so we start by discussing this semigroup. Clearly $S^{-1}$ has nonempty interior if and only if int $S \neq \emptyset$. A consequence of Corollary 4.6 in $[14$ is that $\mathbb{B}\left(S^{-1}\right)$ is the flag manifold dual to $\mathbb{B}(S)$. Since there are imprecisions in the statement and in the proof of that corollary we offer here a version of it.

Proposition 6.1. Take a split-regular $h \in \operatorname{int} S$ and let $A^{+}=\exp \mathfrak{a}^{+}$be the Weyl chamber containing $h$. Then

$$
\mathcal{W}_{A^{-}}\left(S^{-1}\right)=\mathcal{W}_{A^{+}}(S)
$$

where $A^{-}=\left(A^{+}\right)^{-1}$.

Proof. Let $b_{0}$ be the attractor of $h$ and $w_{0}$ the principal involution with respect to $\mathfrak{a}^{+}$. We have $w_{0} A^{+}=A^{-}$and that $w_{0} b_{0}$ is the repeller of $h$ that is the attractor of $h^{-1}$. Let $C$ and $C^{-}$be the invariant control set for $S$ and $S^{-1}$ in $\mathbb{B}$, respectively. By definition $w \in \mathcal{W}_{A^{+}}(S)$ if and only if $D(w)=C$. This means that $w b_{0} \in C$ because $w b_{0}$ is the $w$-fixed-point of $h$ and hence $w b_{0} \in D(w)$. By Theorem 4.5 in [14] $D\left(w w_{0}\right)=D\left(w_{0}\right)$. In fact, this theorem ensures that $\mathcal{W}_{A^{+}}(S) w w_{0}=\mathcal{W}_{A^{+}}(S) w_{0}$ is a consequence of $w \in \mathcal{W}_{A^{+}}(S)$. Since $w_{0}$ is the principal involution $D\left(w_{0}\right)$ is the minimal control set, which is given by $C_{0}^{-}$. Then we get from $D\left(w w_{0}\right)=D\left(w_{0}\right)$ that $w w_{0} b_{0} \in C^{-}$. On the other hand $w w_{0} b_{0}=w\left(w_{0} b_{0}\right)$ is the $w$-fixed-point for $h^{-1}$ because $w_{0} b_{0}$ is its attractor. Hence $w w_{0} b_{0} \in C^{-}$implies that $w \in \mathcal{W}_{A^{-}}\left(S^{-1}\right)$.

Therefore we have $\mathcal{W}_{A^{+}}(S) \subset \mathcal{W}_{A^{-}}\left(S^{-1}\right)$. The reverse inclusion follows from this after remarking that $S=\left(S^{-1}\right)^{-1}$ and $A^{+}=\left(A^{-}\right)^{-1}$.

From this proposition we can define $\mathbb{B}(S)$ and $\mathbb{B}\left(S^{-1}\right)$ by taking the same Weyl chamber $A^{+}$as reference. In doing this it emerges that $\mathbb{B}\left(S^{-1}\right)$ is the dual of $\mathbb{B}(S)$.

Take a split-regular $h \in \operatorname{int} S$ and assume without loss of generality that $h \in$ $A^{+}$. If $\Sigma$ is the associated simple system of roots, then $\mathcal{W}_{A^{+}}(S)$ is generated by reflections with respect to the subset $\Theta(S) \subset \Sigma$. By formula (6.1) $\mathcal{W}_{A^{-}}\left(S^{-1}\right)$ is generated by the same set of reflections. However by definition of $\mathcal{W}_{A^{-}}\left(S^{-1}\right)$ we must look at the generators of this subgroup in the subsets of $-\Sigma$. This is of course $-\Theta$. Hence the parabolic subalgebra associated to $\mathcal{W}_{\mathfrak{a}^{-}}\left(S^{-1}\right)$ is

$$
\mathfrak{p}_{\Theta}^{-}=\theta(\mathfrak{p})+\mathfrak{n}^{+}(\Theta)
$$

where $\mathfrak{n}^{+}(\Theta)$ is the subalgebra spanned by $\mathfrak{g}_{\alpha}$ with $\alpha \in-\langle-\Theta\rangle=\langle\Theta\rangle$ and $\mathfrak{p}$ is the standard minimal parabolic subalgebra. Then $B\left(S^{-1}\right)=G / P_{\Theta}^{-}$. Now $w_{0}(-\Theta)=\iota(\Theta)$ and $w_{0} \mathfrak{p}_{\Theta}^{-}=\mathfrak{p}_{\iota \Theta}$ where

$$
\mathfrak{p}_{\iota \Theta}=\mathfrak{p}+\mathfrak{n}^{-}(\iota(\Theta))
$$


and $\mathfrak{n}^{-}(\iota(\Theta))$ is spanned by $\mathfrak{g}_{-\alpha}$ with $\alpha \in\langle\iota(\Theta)\rangle$. Hence $\mathbb{B}\left(S^{-1}\right)=\mathbb{B}_{\iota(\Theta)}$ the dual of $\mathbb{B}(S)=\mathbb{B}_{\Theta}$. Summarizing

Proposition 6.2. The flag $\mathbb{B}\left(S^{-1}\right)$ is the dual to $\mathbb{B}(S)$.

Returning to the maximal semigroups, it follows easily from this proposition that $S$ is $\Theta$-maximal if and only if $S^{-1}$ is $\Theta^{*}$-maximal. In fact, If $T \supset S^{-1}$ is a semigroup of parabolic type $\Theta^{*}$, then $S \subset T^{-1}$ and $T^{-1}$ is of parabolic type $\Theta$. Hence $S=T^{-1}$ showing that $S^{-1}$ is $\Theta^{*}$-maximal if $S$ is $\Theta$-maximal. Having this fact in mind we can describe $S^{-1}$ as a compression semigroup.

Proposition 6.3. Let $S$ be a $\Theta$-maximal semigroup and denote by $C$ its invariant control set in $\mathbb{B}_{\Theta}$. Then the invariant control set of $S^{-1}$ in $\mathbb{B}_{\Theta^{*}}$ is $\operatorname{cl}\left(C^{*}\right)$. Moreover $S^{-1}$ is the compression semigroup $S_{\mathrm{cl}\left(C^{*}\right)}$.

Proof. By the $S$-invariance of $C$ it follows that $C^{*}$ is invariant under $S^{-1}$ (see Corollary 3.11). Hence $\operatorname{cl}\left(C^{*}\right)$ is $S^{-1}$-invariant so that $S \subset S_{\operatorname{cl}\left(C^{*}\right)}$. But $\operatorname{cl}\left(C^{*}\right)=$ $(\operatorname{int} C)^{*}$ hence by Theorem 5.4, $S_{\operatorname{cl}\left(C^{*}\right)}$ is $\Theta^{*}$-maximal. The equality $S^{-1}=S_{\operatorname{cl}\left(C^{*}\right)}$ follows then by the $\Theta^{*}$-maximality of $S^{-1}$.

This proposition allows the determination of the minimal control sets of the maximal semigroup $S=S_{C}$. In fact, in any flag manifold the minimal control set of $S$ is the set of transitivity of the invariant control set of $S^{-1}$. Keeping the above notations, the invariant control set of $S^{-1}$ in $\mathbb{B}_{\Theta^{*}}$ is $D=\operatorname{cl}\left(C^{*}\right)$ and its set of transitivity is $D_{0}=\operatorname{int}\left(\operatorname{cl}\left(C^{*}\right)\right)$, which contains $C^{*}$ densely. Moreover, let $\pi: \mathbb{B} \rightarrow \mathbb{B}_{\Theta^{*}}$ be the fibration from the maximal flag manifold. Then $\pi^{-1}(D)$ is the invariant control set for $S^{-1}$ in $\mathbb{B}$ and its core is $\pi^{-1}\left(D_{0}\right)$ (see Proposition 4.1). Also, if $\mathbb{B}_{\Theta^{\prime}}$ is any flag manifold, the projection $\pi_{\Theta^{\prime}}: \mathbb{B} \rightarrow \mathbb{B}_{\Theta^{\prime}}$ maps control sets and their cores into control sets and cores respectively. Hence the minimal control set for $S$ in $\mathbb{B}_{\Theta^{\prime}}$ is $\pi_{\Theta^{\prime}}\left(\pi^{-1}\left(D_{0}\right)\right)$. Since the projections between the flag manifolds and their inverse images preserve closures and interiors of subsets we get the minimal control set as the interior of the closure of $\pi_{\Theta^{\prime}}\left(\pi^{-1}\left(C^{*}\right)\right)$.

The subset $\pi_{\Theta^{\prime}}\left(\pi^{-1}\left(C^{*}\right)\right)$ is easily described in terms of incidence of parabolic subalgebras and their nilradicals: Think of a point $x \in \mathbb{B}_{\Theta^{*}}$ as being the nilradical of the corresponding parabolic subalgebra. Viewing the elements of $\mathbb{B}$ as minimal parabolic subalgebras the fiber $\pi^{-1}\{x\}$ is the set of minimal parabolic subalgebras containing $x$. On the other hand, if $y \in \mathbb{B}$ then $\pi_{\Theta^{\prime}}(y)$ is the only parabolic subalgebra in $\mathbb{B}_{\Theta^{\prime}}$ containing $y$. Hence the parabolic subalgebras in $\pi_{\Theta^{\prime}}\left(\pi^{-1}\{x\}\right)$ contain $x$. Reciprocally if $z \in \mathbb{B}_{\Theta^{\prime}}$ is a parabolic subalgebra containing $x$, then there exists a minimal parabolic subalgebra $y \in \pi_{\Theta^{\prime}}^{-1}\{z\}$ containing $x$ so that $z \in$ $\pi_{\Theta^{\prime}}\left(\pi^{-1}\{x\}\right)$. Therefore $\pi_{\Theta^{\prime}}\left(\pi^{-1}\{x\}\right)$ is the set of parabolic subalgebras in $\mathbb{B}_{\Theta^{\prime}}$ containing the nilradical $x$. Thus from the previous paragraph we can state:

Proposition 6.4. Let $S=S_{C}$ be a $\Theta$-maximal semigroup. Given a flag $\mathbb{B}_{\Theta^{\prime}}$ denote by $\overline{C^{*}}$ the set of parabolic subalgebras in $\mathbb{B}_{\Theta^{\prime}}$ containing the nilradical of the parabolic subalgebras in $C^{*} \subset \mathbb{B}_{\Theta^{*}}$. Then the minimal control set of $S$ in $\mathbb{B}_{\Theta^{\prime}}$ is int $\left(\mathrm{cl}\left(\overline{C^{*}}\right)\right)$.

We mention in passing that the other control sets, or more precisely their cores, are determined from the invariant and the minimal control sets. This is true not only for maximal semigroups but for an arbitrary semigroup $S$ with nonempty interior. The idea is that for any control $D$ of $S$ there exists a control set $D^{-}$of $S^{-1}$ such that $\left(D^{-}\right)_{0}=D_{0}$. The intersection of their domains of attraction (under 
the actions of $S$ and $S^{-1}$ respectively) is $D_{0}$. Now, in [13] it was proved that the domain of attraction of a control set $D(w)$ of $S$ is built from the minimal control set and an algebraic property of $w$, namely its minimal decomposition as a product of simple reflections. In a symmetric way the domain of attraction of $D(w)^{-}$depends only on $w$ and the minimal control set of $S^{-1}$, that is, the invariant control set of $S$. With this construction it is possible to describe the cores of the control sets by incidence of parabolic subalgebras. Since this is not specific for maximal semigroups we leave out the details.

\subsection{Maximal semigroups containing a given semigroup.}

6.2.1. General semigroups. As mentioned above any semigroup with nonvoid interior in a topological group is contained in a maximal one. This very general fact can be improved in our context by means of Theorem 5.4. Starting with a semigroup $S$ of parabolic type $\Theta$ let $C$ be its invariant control set in $\mathbb{B}_{\Theta}$. Then $\cos _{\mathcal{B}}(C)$ is $S$-invariant and the arguments in the proof of Proposition 5.2 ensure that $S$ is contained in the $\Theta$-maximal semigroup $S_{K}$ where $K=\operatorname{cl}\left(\operatorname{int}\left(\cos _{\mathcal{B}}(C)\right)\right)$. Also, if $\Theta \subset \Theta^{\prime}$ then the projection $\pi: \mathbb{B}_{\Theta} \rightarrow \mathbb{B}_{\Theta^{\prime}}$ is defined and the same argument applied to $\pi(C)$ instead of $C$ shows that $S$ is contained in a $\Theta^{\prime}$-maximal semigroup. In particular we recover the general result that there exists a maximal semigroup containing $S$.

In general a semigroup $S$ of parabolic type $\Theta$ can be contained in several $\Theta^{\prime}$ maximal semigroups if $\Theta \subset \Theta^{\prime}$, according to the $\mathcal{B}$-convex sets left invariant by $S$. The following statement exhibits a situation where uniqueness of the maximal semigroup containing $S$ holds.

Proposition 6.5. Let $S=S_{C}$ be a $\Theta$-maximal semigroup with $C=\operatorname{cl}(\operatorname{int} C) a$ $\mathcal{B}$-convex set. Suppose that for $\Theta \subset \Theta^{\prime}, \pi(C)$ is $\mathcal{B}$-convex in $\mathbb{B}_{\Theta^{\prime}}$. Then $S_{\pi(C)}$ is the only $\Theta^{\prime}$-maximal semigroup containing $S$.

Proof. By Theorem 5.4 it follows that $C$ is the invariant control set of $S$ in $\mathbb{B}_{\Theta}$ hence the $S$-invariant control set in $\mathbb{B}_{\Theta^{\prime}}$ is $\pi(C)$. In particular $\pi(C)$ is $S$-invariant so that $S \subset S_{\pi(C)}$. By assumption $\pi(C)$ is $\mathcal{B}$-convex. Moreover, int $(\pi(C))$ is dense in $\pi(C)$ because $\pi$ is an open map. Applying Theorem 5.4 again it follows that $S_{\pi(C)}$ is indeed $\Theta^{\prime}$-maximal. Now let $T$ be a $\Theta^{\prime}$-maximal semigroup containing $S$. Then the invariant control set of $T$ in $\mathbb{B}_{\Theta^{\prime}}$, say $D$, contains $\pi(C)$. Of course $T=S_{\pi(C)}$ if $D=\pi(C)$. On the other hand the arguments in the proof of Proposition 5.3 show that $D \neq \pi(C)$ contradicts the assumption that $T$ is of parabolic type $\Theta^{\prime}$.

6.2.2. Lie semigroups. Let $S$ be an infinitesimally generated semigroup of parabolic type $\Theta$, and denote by $C$ its invariant control set in $\mathbb{B}_{\Theta}$. Since $S$ is connected, $C$ is also connected. Suppose that $T$ is a maximal semigroup of parabolic type $\Theta^{\prime}$, with $\Theta \subset \Theta^{\prime}$ and $S \subset T$. Denote by $D$ and $C_{1}$ the invariant control set of $T$ and $S$ in $\mathbb{B}_{\Theta^{\prime}}$, respectively. Of course, $C_{1} \subset D$ and $C_{1}$ is connected.

Proposition 6.6. $D$ is connected.

Proof. Take $h \in \operatorname{int} S$, then $h \in \operatorname{int} T$. Denote by $b$ the attractor of $h$ in $\mathbb{B}_{\Theta^{\prime}}$ and by $\sigma$ the corresponding stable manifold. We have $b \in \operatorname{int} C_{1}$ and $D \subset \sigma$. Hence for all $x \in D, h^{n} x \in \operatorname{int} C_{1}$ for some large $n$. But $S$ is infinitesimally generated, so that there exists a continuous path $g_{t} \in S, t \in[0, T]$, such that $g_{0}=1$ and $g_{T}=h^{n}$. Since $D$ is $S$-invariant, $g_{t} x \in D$ for all $t \in[0, T]$. Hence there exists a continuous path connecting $x$ to $C_{1}$, implying that $D$ is connected. 


\subsection{Examples.}

6.3.1. Total positivity. A square matrix with real entries is said to be totally positive provided its minors of all orders are nonnegative numbers. It is well known that the set $T$ of totally positive matrices in $\mathrm{Sl}(n, \mathbb{R})$ is a semigroup with nonvoid interior. We consider here the maximality properties of a semigroup slightly larger than $T$ : An $n \times n$ matrix is said to be sign-regular if for every $k=1, \ldots, n-1$, its minors of order $k$ have the same sign. The semigroup $\bar{T}$ of sign-regular matrices clearly contains $T$. It is a compression semigroup as the following constructions shows.

Let $\Lambda_{k}=\bigwedge^{k} \mathbb{R}^{n}$ be the $k$-fold exterior product of $\mathbb{R}^{n}$. The Grassmannian $\operatorname{Gr}_{k}(n)$ embeds into the projective space of $\Lambda_{k}$ as the set of lines spanned by the decomposable elements. Analogously the Grassmannian $\mathrm{Gr}_{k}^{+}(n)$ of oriented $k$-dimensional subspaces, which is a two-fold covering of $\mathrm{Gr}_{k}(n)$ embeds in a sphere of $\Lambda_{k}$. For $g \in \mathrm{Sl}(n, \mathbb{R})$ denote also by $g$ the induced linear map of $\Lambda_{k}$. Both Grassmannians $\operatorname{Gr}_{k}(n)$ and $\mathrm{Gr}_{k}^{+}(n)$ are invariant under $g \in \mathrm{Sl}(n, \mathbb{R})$.

Let $\beta_{1}=\left\{e_{1}, \ldots, e_{n}\right\}$ be the standard basis of $\mathbb{R}^{n}$ and $\beta_{k}=\left\{e_{I}=e_{i_{1}} \wedge \cdots \wedge e_{i_{k}}\right\}$ where $I=\left(i_{1}<\cdots<i_{k}\right)$ the basis induced in $\Lambda_{k}$. This basis is orthonormal with respect to the inner product $\langle\cdot, \cdot\rangle$ in $\Lambda_{k}$ coming from the standard inner product in $\mathbb{R}^{n}$. The positive orthant in $\Lambda_{k}$ is determined by the inequalities $\left\langle e_{I}, \cdot\right\rangle \geq 0$. We denote by $\mathcal{O}_{k}$ its intersection with the oriented $\operatorname{Grassmannian} \mathrm{Gr}_{k}^{+}(n)$ :

$$
\mathcal{O}_{k}=\left\{v \in \operatorname{Gr}_{k}^{+}(n):\left\langle v, e_{I}\right\rangle \geq 0 \text { for all } I\right\} .
$$

Consider the compression semigroup

$$
T_{k}=\left\{g \in \mathrm{Sl}(n, \mathbb{R}): g \mathcal{O}_{k} \subset \mathcal{O}_{k}\right\}
$$

Since the $k$-minors of $g$ are the entries $\left\langle g e_{I}, e_{J}\right\rangle$ of the matrix of $g_{k}$ with respect to $\beta_{k}$, it follows that $g \in T_{k}$ if and only if all its minors of order $k$ are nonnegative. Hence

$$
T=T_{1} \cap \cdots \cap T_{n-1} .
$$

Put $C_{k}=\pi\left(\mathcal{O}_{k}\right)$ where $\pi: \operatorname{Gr}_{k}^{+}(n) \rightarrow \operatorname{Gr}_{k}(n)$ is the canonical projection and set

$$
\bar{T}_{k}=\left\{g \in \mathrm{Sl}(n, \mathbb{R}): g C_{k} \subset C_{k}\right\} .
$$

It is easily checked that $g \in \bar{T}_{k}$ if and only if either $g \in T_{k}$ or all the $k$-minors of $g$ are negative. Hence

$$
\bar{T}=\bar{T}_{1} \cap \cdots \cap \bar{T}_{n-1} .
$$

Now we verify that $C_{k}$ is $\mathcal{B}$-convex. This will be a consequence of

Lemma 6.7. For $V \notin \operatorname{int}\left(C_{k}\right)$ let $V^{\perp}$ be its orthocomplement in $\mathbb{R}^{n}$. Then $V^{\perp} \notin$ $C_{k}^{*}$, i.e., there exists $W \in C_{k}$ with $\operatorname{dim}\left(W \cap V^{\perp}\right) \geq 1$.

Proof. Take a basis $\left\{v_{1}, \ldots, v_{k}\right\}$ of $V$ and let $v=v_{1} \wedge \cdots \wedge v_{k}$ be the associated decomposable vector in $\Lambda_{k}$.

If $V$ is in the boundary of $C_{k}$, then $\left\langle v, e_{I}\right\rangle=0$ for some basic element $e_{I}=$ $e_{i_{1}} \wedge \cdots \wedge e_{i_{k}}$. Put $E_{I}=\operatorname{span}\left\{e_{i_{1}}, \ldots, e_{i_{k}}\right\}$. Then $\left\langle v, e_{I}\right\rangle=0$ is equivalent to $\operatorname{dim}\left(E_{I} \cap V^{\perp}\right) \geq 1$. Since $E_{I} \in C_{k}$ this shows the lemma in case $V \in C_{k}$.

Assume that $V \notin C_{k}$ and consider the continuous map

$$
f_{v}: w \in \mathrm{Gr}_{k}^{+}(n) \longmapsto\langle v, w\rangle \in \mathbb{R} .
$$

By definition of $C_{k}$ it follows that $v \notin \pm \mathcal{O}_{k}$ so that there are indices $I, J$ such that $\left\langle v, e_{I}\right\rangle>0$ and $\left\langle v, e_{J}\right\rangle<0$. Let $A$ be the subgroup of diagonal matrices with 
positive eigenvalues. This subgroup is connected and leaves invariant the orthant $\mathcal{O}_{k}$. Moreover it is easy to find $g, h \in A$ and $z \in \mathcal{O}_{k}$ such that $g^{i} z \rightarrow e_{I}$ and $h^{i} z \rightarrow e_{J}$ as $i \rightarrow+\infty$. Hence $f_{v}$ assumes positive and negative values in $A z$, implying that there exists $w=w_{1} \wedge \cdots \wedge w_{k}$ in $\mathcal{O}_{k}$ such that $\langle v, w\rangle=0$. Put $W=\operatorname{span}\left\{w_{1}, \ldots, w_{k}\right\}$. Then $\langle v, w\rangle=0$ means that $\operatorname{dim}\left(W \cap V^{\perp}\right) \geq 1$. Since $W \in C_{k}$ this shows the lemma.

This lemma shows immediately that if an $(n-k)$-dimensional subspace $U$ belongs to $C_{k}^{*}$, then its orthocomplement $U^{\perp}$ belongs to int $\left(C_{k}\right)$. Reciprocally, take $V, W \in \operatorname{int}\left(C_{k}\right)$ and choose bases $\left\{v_{1}, \ldots, v_{k}\right\}$ and $\left\{w_{1}, \ldots, w_{k}\right\}$ of $V$ and $W$ respectively such that $v=v_{1} \wedge \cdots \wedge v_{k}$ and $w=w_{1} \wedge \cdots \wedge w_{k}$ are in $\operatorname{int}\left(\mathcal{O}_{k}\right)$. Then $\langle v, w\rangle>0$ because $\mathcal{O}_{k}$ is an orthant defined by an orthonormal basis. Hence $V \cap W^{\perp}=0=W \cap V^{\perp}$ so that $V^{\perp}, W^{\perp} \in C_{k}^{*}$. Therefore

$$
C_{k}^{*}=\left\{V^{\perp}: V \in \operatorname{int}\left(C_{k}\right)\right\} .
$$

The above lemma also shows that $V \notin C_{k}^{* *}$ if $V \notin C_{k}$ so that $C_{k}=C_{k}^{* *}$ is $\mathcal{B}$-convex. Therefore,

Proposition 6.8. $\bar{T}_{k}$ is maximal for all $k=1, \ldots, n-1$.

We leave aside further discussions about the semigroup $\bar{T}$, but mention that a similar approach shows for any sequence $\mathbf{r}=\left(r_{1}<\cdots<r_{m}\right)$, the semigroup

$$
\bar{T}_{\mathbf{r}}=\bar{T}_{r_{1}} \cap \cdots \cap \bar{T}_{r_{m}}
$$

is maximal with respect to $\mathbb{F}(\mathbf{r})$. In particular $\bar{T}$ is maximal with respect to the maximal flag manifold.

We refer to Ando [1] for a survey about totally positivity matrices. See also Lusztig [9] and references therein for a generalization to semi-simple groups.

6.3.2. A class of compression semigroups. The following example is a particular instance of the compression semigroups considered by Hilgert and Neeb [7]. Let $Q$ be a quadratic form in $\mathbb{R}^{n}$ with matrix

$$
\left(\begin{array}{cc}
1_{k \times k} & 0 \\
0 & -1_{(n-k) \times(n-k)}
\end{array}\right) .
$$

Denote by $\beta$ the corresponding nondegenerate bilinear form. Let $C \subset \operatorname{Gr}_{k}(n)$ be the set of subspaces where $Q$ is positive semi-definite and consider the compression semigroup $S_{C}$ as a subsemigroup of $\operatorname{Sl}(n, \mathbb{R})$. The continuity of $Q$ ensures that $C=\operatorname{cl}(\operatorname{int} C)$. Moreover, let $U \in \operatorname{Gr}_{n-k}(n)$ be such $Q$ is negative definite on $U$. Then $Q$ is negative definite in any subspace of $U$. This implies that $V \cap U=0$ for all $V \in C$. Hence $C \subset \sigma_{U}$ so that $C$ is admissible and $U \in C^{*}$. Therefore $S_{C}$ has nonempty interior and is of parabolic type $\operatorname{Gr}_{k}(n)$.

Denote by $D \subset \operatorname{Gr}_{n-k}(n)$ the set of subspaces where $Q$ is negative definite. We have just seen that $D \subset C^{*}$ or equivalently $C \subset D^{*}$. We claim that $C=D^{*}$. To check this use the well-known fact that if $W \subset \mathbb{R}^{n}$ is a subspace with $\operatorname{dim} W \leq n-k$ and such that $Q$ is negative definite in $W$ then it extends to a subspace $U \supset W$ with $\operatorname{dim} U=n-k$ and $Q$ negative definite in $U$.

Now suppose that there exists $V \in D^{*}$ such that $Q$ is not positive semi-definite in $V$. Then there exists a subspace $W \subset V$ where $Q$ is negative definite. Since $W$ extends to an element of $D$ this contradicts the fact that $V$ is transversal to every element of $D$. Hence $D^{*} \subset C$ and $C=D^{*}$. 
Therefore $C$ is $\mathcal{B}$-convex which implies that $S_{C}$ is maximal of parabolic type $\operatorname{Gr}_{k}(n)$, and hence maximal in $\mathrm{Sl}(n, \mathbb{R})$.

6.4. Remarks and questions. Although Theorem 5.4 gives an exact characterization of the maximal semigroups in terms of $\mathcal{B}$-convexity it is far from being conclusive for the full understanding of the maximal semigroups. Specially in what concerns specific classes of semigroups, like e.g. connected semigroups, infinitesimally generated semigroups, etc. For deeper insights into the maximal semigroups our results must be followed by a further development of the geometry of the $\mathcal{B}$ convex sets and their compression semigroups. Below we list some natural questions and remarks pointing to this direction.

1. From the work of Lawson [8] one knows that a maximal semigroup $S$ in a solvable group $G$ is total in the sense that $G=S \cup S^{-1}$. This property cannot hold for semigroups in semi-simple groups because of the existence of an open set of compact elements. However one can ask whether a maximal semigroup is total with respect to a flag manifold $\mathbb{B}_{\Theta}$, in the sense that $\mathbb{B}_{\Theta}$ is the union of the $S$-control sets. With this kind of totality the action of $S$ on the flag manifold is completely clear since one knows the action inside the control sets. At this regard we mention that under totality the proof of Proposition 5.3 would be simplified since what is required there is to show that a point outside the invariant control set belongs to the domain of attraction of another control set.

2. If $S$ is connected then its invariant control set (in any homogeneous space of $G$ ) is connected. This suggests the investigation of the compression semigroups $S_{C}$ with $C$ connected and $\mathcal{B}$-convex. In general $S_{C}$ is not connected. This is shown for instance by the compression semigroup in $\mathrm{Sl}(2, \mathbb{R})$ of an interval in the projective line $\mathbb{P}^{1}$. It has two connected components $\pm \mathrm{Sl}^{+}(2, \mathbb{R})$, where $\mathrm{Sl}^{+}(2, \mathbb{R})$ is the semigroup of $2 \times 2$ matrices with positive entries. However $\mathrm{Sl}^{+}(2, \mathbb{R})$ is connected and maximal with this property. Similar facts may occur in general: There might be a class of connected $\mathcal{B}$-convex sets which are invariant control sets of semigroups which are maximal with the property of being connected. This development certainly goes through the study of the connected $\mathcal{B}$-convex sets and the $\mathcal{B}$-convex hull of connected sets, which in general may not be connected. Of course the same kind of questions make sense for $\Theta$-maximal semigroups.

3. Similar remarks apply to the infinitesimally generated semigroups. Here one of the basic questions seems to be a characterization of the maximal semigroups (and corresponding $\mathcal{B}$-convex sets) whose tangent wedge generate a semigroup with the same invariant control set (see D. Mittenhuber 10]).

4. It looks like that Proposition 6.5 can be improved by showing that the projection of a $\mathcal{B}$-convex set is $\mathcal{B}$-convex, at least for large classes of $\mathcal{B}$-convex sets.

\section{REFERENCES}

[1] Ando, T.: Totally positive matrices. Linear Algebra Appl. 90 (1987), 165-219. MR 88b:15023

[2] Colonius, K. and W. Kliemann: "Dynamics and control". Birkhäuser (2000). MR 2001e:93001

[3] Goodman, J.E.: When is a set of lines in space convex? Notices Amer. Math. Soc. 45 (1998), $222-232$. 
[4] Goodman, J.E. and R. Pollack: Foundations of a theory of convexity on affine Grassmann manifolds. Mathematika 42 (1995), 305-328. MR 97a:52002

[5] Hilgert, J., K.H. Hofmann and J. Lawson: "Lie groups, convex cones, and semigroups". Oxford University Press (1989). MR 91b:22008

[6] Hilgert, J. and K.-H. Neeb: "Lie semigroups and their applications". Lecture Notes in Math. 1552, Springer-Verlag (1993). MR 96j:22002

[7] Hilgert, J. and K.-H. Neeb: Maximality of compression semigroups. Semigroup Forum 50 (1995), 205-222. MR 96e:53069

[8] Lawson, J.D.: Maximal semigroups of Lie groups that are total. Proc. Edinburgh Math. Soc. 30 (1987) 479-501. MR 89e:22042

[9] Lusztig, G.: Introduction to total positivity. In "Positivity in Lie Theory: Open Problems" (Eds. J. Hilgert, J.D. Lawson, K.-H. Neeb and E.B. Vinberg). De Gruyter Expositions in Mathematics 26 (1998), 133-145. MR 99h:20077

[10] Mittenhuber, D.: Compression semigroups in semisimple Lie groups: a direct approach. In "Positivity in Lie Theory: Open Problems" (Eds. J. Hilgert, J.D. Lawson, K.-H. Neeb and E.B. Vinberg). De Gruyter Expositions in Mathematics 26 (1998), 165-183. MR 99j:22009

[11] do Rocio, O.G. and L.A.B. San Martin: Semigroups in lattices of solvable Lie groups. J. Lie Theory 51 (1995), 179-202. MR 97d:22007

[12] San Martin L.A.B.: Invariant control sets on flag manifolds. Math. Control Signals Systems 6 (1993), 41-61. MR 96i:93020

[13] - Order and domains of attractions of control sets in flag manifolds. J. Lie Theory 8 (1998), 335-350. MR 99j:93024

[14] San Martin, L.A.B. and P.A. Tonelli: Semigroup actions on homogeneous spaces. Semigroup Forum 50 (1995), 59-88. MR 97a:22008

[15] Warner, G.: "Harmonic analysis on semi-simple Lie groups I". Springer-Verlag (1972). MR 58:16979

Instituto de Matemática, Universidade Estadual de Campinas, Cx.Postal 6065, 13083970 CAMPINAS SP, BRASIL

E-mail address: smartin@ime.unicamp.br 